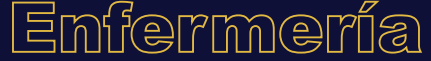

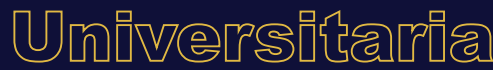

\section{Funcionamiento familiar, sobrecarga y calidad de vida del cuidador del adulto mayor con dependencia funcional}

Family dynamics, overload, and quality of life among caregiver of functionally dependent older adults

\section{Funcionamento familiar, sobrecarga e qualidade de vida no cuidador do idoso com dependência funcional}

M.E. Salazar-Barajas ${ }^{\text {a1 }}$, E.G. Garza-Sarmiento ${ }^{\text {b1 }}$, S.N. García-Rodríguez ${ }^{\mathrm{cl}}$, P.Y. Juárez-Vázquez ${ }^{\mathrm{d} \text {, }}$, J.L. Herrera-Herrera ${ }^{\mathrm{e}}$, T. Duran-Badillo ${ }^{\mathrm{fi}}$

ORCID:

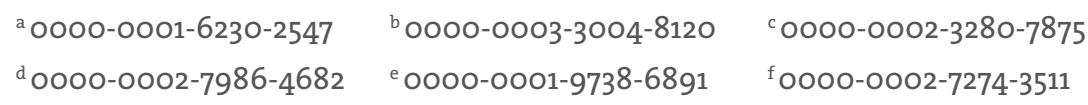

Introducción: Ser cuidador de personas con dependencia funcional conlleva el riesgo de presentar deterioro de la calidad de vida, esto puede deberse a que la prolongación del cuidado altera la dinámica familiar y genera sobrecarga en el cuidador. 
Objetivo: 1) Identificar la relación entre funcionamiento familiar y calidad de vida del cuidador del adulto mayor y 2) Determinar la sobrecarga y calidad de vida del cuidador del adulto mayor.

Metodología: Estudio descriptivo y correlacional, muestreo por bola de nieve, la muestra conformada por 86 cuidadores de un centro comunitario. Para las mediciones se utilizó el índice de Barthel, el Test de APGAR Familiar, la prueba de Zarit y la Escala de Calidad de Vida WHOOOLBREF. Se aplicó la Prueba de Kolmogorov Smirnov y Prueba de Correlación de Spearman.

Resultados: El 11.6\% de los adultos mayores presentó dependencia total, la percepción de disfuncionalidad familiar grave representó el 3.5\%. Los cuidadores con sobrecarga intensa fueron el 32.6\%, el 8.1\% percibió la calidad de vida deficiente. A mayor funcionamiento familiar mayor calidad de vida del cuidador ( $p<.05$ ) y a mayor sobrecarga, menor calidad de vida del cuidador ( $p<.05)$ en todas las dimensiones.

Conclusiones: Se observó que la sobrecarga y funcionalidad familiar están relacionadas con la calidad de vida del cuidador, por lo que se convierten en un escenario disciplinar que plantea la posibilidad de diseñar estrategias de intervención, centradas en la sobrecarga y el funcionamiento de la familia para el mejoramiento de la calidad de vida del cuidador.

Palabras clave: Relaciones familiares; cuidadores; calidad de vida; anciano; México.

\section{ABSTRACT}

Introduction: The prolonged provision of healthcare to functionally dependent persons can result in the deterioration on the caregiver's quality in terms of family dynamics and overload. Objectives: 1) To identify the relationship between the family dynamics and the quality of life of the caregiver of older adults; and 2) to estimate the quality of life and the overload of the caregiver of older adults.

Methodology: This is a descriptive and correlational study with a snowball-derived sample of 86 caregivers in a communitarian center. The corresponding measures were estimated using the Barthel Index, the Family APGAR Test, the Zarit Test, and the WHOOOL-BREF Quality of Life Scale. The Kolmogorov-Smirnov and Spearman Correlation tests were calculated.

Results: $11.6 \%$ of the older adults being cared showed total dependency. $3.5 \%$ of the caregiver's sample perceived having a severe family dysfunction. $32.6 \%$ of the sample were found having heavy overloads. $8.1 \%$ of the sample perceived having poor quality of life. In all dimensions, the better the family dynamics were, the better the quality of life of the caregivers was ( $p<.05)$, and the heavier the overload was, the worse the quality of life of the provider was ( $p<.05)$. Conclusions: It was noted that both, overload and family dynamics, are related to the quality of life of the caregivers of older adults; therefore, the design of intervention strategies should be focused on the overload and family dynamics of these professionals in order to improve their quality of life.

Keywords: Family relationships; caregivers; quality of life; aged; Mexico.

\section{RESUMO}

Introdução: Ser cuidador de pessoas com dependência funcional implica o risco de apresentar deterioro da qualidade de vida, isto pode dever-se a que o prolongamento do cuidado altera a dinâmica familiar e gera sobrecarga no cuidador.

Objetivo: 1) Identificar a relação entre funcionamento familiar e qualidade de vida do cuidador do idoso e 2) Determinar a sobrecarga e qualidade de vida no cuidador do idoso. 
Metodologia: Estudo descritivo e correlacional, amostragem por bola de neve, a amostragem conformada por 86 cuidadores de um centro comunitário. Para as medições utilizou-se o índice de Barthel, o Teste de APGAR Familiar, a prova de Zarit e a Escala de Qualidade de Vida WHOQOL-BREF. Aplicou-se a prova de Kolmogorov Smirnov e Prova de Correlação de Spearman.

Resultados: O 11.6\% dos idosos apresentou dependência total, a percepção de disfuncionalidade familiar grave representou o 3.5\%. Os cuidadores com sobrecarga intensa foram o 32.6\%, o 8.1\% percebeu a qualidade de vida deficiente. Quanto maior funcionamento familiar, maior qualidade de vida do cuidador ( $\mathrm{p}<.05$ ) e quanto maior sobrecarga, menor qualidade de vida do cuidador ( $\mathrm{p}<.05)$ em todas as dimensões.

Conclusões: Observou-se que a sobrecarga e funcionalidade familiar estão relacionadas com a qualidade de vida do cuidador, pelo que se tornam em um cenário disciplinar que envolve a possibilidade de desenhar estratégias de intervenção, centradas na sobrecarga e o funcionamento da família para o melhoramento da qualidade de vida do cuidador.

Palavras chave: Relações familiares; cuidadores; qualidade de vida; idoso; México.

\section{INTRODUCCIÓN}

Los avances tecnológicos en la salud han intervenido de manera considerable en el aumento de la esperanza de vida de la población y en la regulación de la fecundidad. A medida que los individuos acumulan años de vida se presentan cambios moleculares y celulares, como resultado de esto se desencadena un descenso gradual de las capacidades físicas y mentales, que resultan más evidentes en las personas mayores de 65 años. Actualmente en la población mexicana existen alrededor de 13 millones de adultos mayores ${ }^{1}$, estas cifras van en incremento y se espera que en el 2050 dicho grupo corresponda al $28.7 \%$ de la población total ${ }^{2}$.

A medida que la persona envejece se aumenta el riesgo de que esta enferme o sufra afecciones físicas, y con ello, la necesidad de ser cuidada por una o más personas 3 . La persona con necesidad de ayuda para realizar las actividades cotidianas es considerada dependiente y la ayuda comúnmente es otorgada por la familia4.

A la persona que cuida o asiste a otra persona durante un proceso de enfermedad, discapacidad o minusvalía se le llama cuidador. Por lo general los cuidadores son mujeres y entre ellas se distinguen sus familiares y preferentemente las hijas5; quienes no reciben recompensa económica por la actividad que realizan. Según la extensión del cuidado y el grado de responsabilidad que el cuidador brinda, se han catalogado como primarios y secundarios; los primeros tienen mayor sobrecarga, son los más cercanos y están encargados de organizar todas las tareas del cuidado6.

La sobrecarga desde la perspectiva de la teoría del estrés es considerada multidimensional con características objetivas y subjetivas, objetiva porque trasciende a los cambios que realiza el cuidador y subjetiva por aquellas reacciones de tipo emocional frente a las demandas del cuidado7.

Los problemas físicos como resultado de una sobrecarga en el cuidador pueden ser cefalea, astenia, fatiga crónica y alteración del ciclo del sueño. Otras manifestaciones son de tipo emocional como los estados depresivos y de ansiedad; también existen problemas de tipo social, entre ellos destaca el alejamiento de amigos y conocidos. Además, de todo lo anterior la literatura señala que la mayoría de los cuidadores no buscan ayuda de los profesionales de salud, ejemplo de ello es cuando estos enferman al postergar su cuidado y priorizar los problemas de su familiar dependiente ${ }^{8}$. 
Tanto los problemas físicos, psicológicos y sociales del cuidador generan cambios en la dinámica familiar con impacto en sus relaciones afectivas, dando como resultado la alteración del funcionamiento familiar. La funcionalidad familiar es el conjunto de relaciones interpersonales que se generan en el interior de cada familia y que le confieren identidad propia. Según el modelo teórico de Chagoya L, la funcionalidad de la familia se mide por la capacidad para resolver las crisis que se le presentan a la familia, y las formas en que expresan sus afectos9.

Un buen funcionamiento familiar incluye la adaptación como punto de partida entre cada uno de los integrantes de la familia, la capacidad para resolver los problemas que se generan como resultado de las demandas del cuidado, la participación requerida de cada uno de ellos, el crecimiento que involucra el aspecto emocional y físico con el logro de los objetivos, el afecto considerado esencial entre los integrantes de la familia y el dependiente y por último, la resolución de problemas que involucra recursos económicos y el factor tiempo ${ }^{10}$.

En el cuidado de la persona dependiente cada aspecto es muy importante para el funcionamiento familiar y posiblemente, la participación de cada integrante de la familia es el aspecto más esperado, pero al no involucrarse todos sus miembros, la responsabilidad recae en una sola persona, alterando así su nivel de carga y calidad de vida como consecuente ${ }^{11}$.

La calidad de vida se puede evaluar a partir de la percepción que un individuo tiene de su lugar en la existencia, en el contexto de la cultura y del sistema de valores en los que vive y en relación con sus objetivos, sus expectativas, sus normas, sus inquietudes, valores e intereses. La calidad de vida puede estar influenciada además por aspectos como la salud, la alimentación, la educación, el trabajo, la vivienda, la seguridad social, el vestido, el ocio y los derechos humanos 3 .

Se ha reportado que los cuidadores no reconocen el impacto de la sobrecarga sobre su estado de salud y calidad de vida, por lo que su condición empeora debido a que para ellos su prioridad es el cuidado de la persona dependiente. En nuestro contexto, los adultos mayores son cuidados en casa por integrantes de la familia, lo que favorece el surgimiento de limitaciones o alteración de la calidad de vida de los cuidadores y riesgos para la salud ${ }^{12}$.

La literatura reporta hallazgos sobre la calidad de vida de los cuidadores y como estos presentan estrés crónico, sufrimiento, aislamiento, abandono, conflicto de roles, de pareja y problemas económicos a consecuencia de la sobrecarga. También se ha indicado la implicación de la cronicidad y cómo el hacerse cargo de la persona enferma por periodos prolongados, genera en la salud física del cuidador problemas de salud muy diversos como fatiga, dolor, cambios en el apetito y en el dormir, mareo, náuseas, dolor de cabeza, de espalda, de piernas, sensación de cansancio y pérdida de energía. Otras alteraciones son en el patrón de sueño, astenia y fatiga crónica, alteraciones osteomusculares y cardiovasculares ${ }^{13}$.

Un estudio realizado en Brasil muestra que el 77.6\% de los cuidadores son hijos de adultos mayores y que la edad avanzada de los cuidadores es motivo de preocupación, ya que puede influir directamente en el riesgo de sobrecarga ${ }^{12}$. Otros autores reportan que los cuidadores presentan $18.1 \%$ de algún tipo de sobrecarga ${ }^{14}$. En México se ha estudiado la sobrecarga en cuidadores y según el test de Zarit, el 56\% de los cuidadores presentó algún grado de sobrecarga. Aunado a lo anterior, a mayor número de horas es mayor el nivel de sobrecarga ${ }^{15}$. Otros autores concluyeron en su estudio que entre mayor es la dependencia del adulto mayor, mayor es el nivel desobrecarga ${ }^{16}$.

A su vez, los estudios referentes al funcionamiento familiar han encontrado que el $62.5 \%$ de las familias tenían una alta funcionalidad, mientras que el $37 \%$ reportaron una funcionalidad familiar severamente disfuncional ${ }^{17}$. Es bien sabido que al tener una persona de edad avanzada en casa y 
con una patología crónica, se requiere la participación de la familia en el proceso de atención. Se sugiere que la presencia de la enfermedad hace que las familias presenten fallas de relación entre sus miembros ${ }^{18}$.

Como se ha mostrado, estos estudios han evaluado el nivel de sobrecarga, la relación de esta en función del grado de dependencia del adulto mayor, así como la vinculación de estos factores con la calidad de vida. Sin embargo; no se ha explorado la relación entre el funcionamiento familiar, sobrecarga y calidad de vida del cuidador. Identificar esta relación brindaría elementos respecto a otras condiciones que afectan a los cuidadores, así enfermería podrá proponer programas específicos que promuevan mejor calidad de vida de los cuidadores y el bienestar del adulto mayor. Por todo lo anterior, se propuso realizar un estudio, con el propósito de: 1) Identificar la relación entre funcionamiento familiar y calidad de vida del cuidador del adulto mayor y 2) Determinar la sobrecarga y calidad de vida del cuidador del Adulto Mayor en la ciudad de Matamoros, Tamaulipas, México.

\section{METODOLOGÍA}

Se realizó un estudio descriptivo, correlacional, en una población de cuidadores de adultos mayores con dependencia funcional que son atendidos en un Centro de Salud Comunitario en Matamoros, Tamaulipas. Debido a que no se contaba con un marco muestral, se realizó un muestreo no probabilístico por bola de nieve en el segundo semestre del año 2018, en el que se identificaron 86 cuidadores que por lo menos tuvieran seis meses al cuidado del adulto mayor dependiente.

La dependencia se evaluó con el índice de Barthel pues es un instrumento fácil de aplicar, con demostrada validez y fiabilidad, que mide la capacidad funcional para realizar las actividades de la vida diaria con una puntuación que oscila entre cero y cien puntos ${ }^{19}$. Se califica como dependiente total cuando se obtiene menos de 21 puntos, de 21 a 60 puntos con dependencia severa, de 61 a 90 puntos dependencia moderada, de 91 a 99 puntos dependencia escasa y 100 puntos, total independencia del adulto mayor ${ }^{20}$.

El funcionamiento familiar se midió con el Test de APGAR familiar compuesto por cinco reactivos. Cada respuesta tiene un puntaje que oscila entre los o y 2 puntos, de acuerdo con la siguiente calificación: o Casi Nunca, 1 Algunas veces y 2 Casi siempre. El índice de la puntuación del instrumento es entre cero y diez puntos, de cero a tres se califica como disfunción grave; de cuatro a seis, disfunción moderada; y de siete a diez, funcionalidad normal. Se ha demostrado validez y confiabilidad del APGAR familiar para su uso en adultos. Los índices de correlación oscilan entre .71 y .83 para diversos contextos ${ }^{10}$.

La sobrecarga del cuidador se midió con la prueba de Zarit, que consta de 22 ítems, en la que cada uno se califica con una frecuencia que va desde 1(nunca), 2 (rara vez), 3 (algunas veces), 4 (Bastantes veces) y 5 (casi siempre), luego este se suma obteniendo como resultado entre 22 a 110 puntos. Menos de 46 puntos se califica sin sobrecarga, de 46 a 55 puntos sobrecarga leve) y 56 o más puntos sobrecarga intensa ${ }^{21}$. Este instrumento ha sido validado, el alfa de Cronbach reportado para esta escala es de 0.91 con un coeficiente de correlación de Pearson $=0.86$ para fiabilidad test-retest ${ }^{22}$.

Para la calidad de vida se utilizó la Escala de Calidad de Vida WHOOOL-BREF, que contiene 26 preguntas, las dos primeras indagan la percepción del individuo sobre su calidad de vida y salud respectivamente; las 24 restantes evalúan cuatro dimensiones específicas de la calidad de vida: física (ítems 3, 4, 10, 15, 16, 17 y 18), psicológica (ítems 5, 6, 7, 11, 19 y 26), social (ítems 20, 21 y 22) y ambiental (ítems 8, 9, 12, 13, 14, 23, 24 y 25). Cada pregunta tiene opciones de respuesta tipo Likert de 1 a 5, mientras más alta sea la puntuación asignada, más alta será la calidad de vida. Se invierten los puntajes 
de los ítems negativos ( 3,4 y 26) y los puntajes totales obtenidos se convierten en una escala de o a 100 para poder establecer comparaciones entre los dominios. La percepción de la calidad de vida y la salud se clasificó en deficiente cuando resultó o y 2.99, aceptable de 3 a 3.99 y alta entre 4 y 5 . Esta es una escala confiable que ha sido utilizada recientemente en población similar ${ }^{23}$. Este test ha demostrado tener buena validez discriminante de contenido y confiabilidad test-retest ${ }^{24} \mathrm{El}$ alfa reportado para este instrumento por cada dimensión es de: físico 0.82, psicológico 0.81, mental 0.80 y social $0.68^{25}$.

Antes de aplicar los instrumentos se contó con la autorización de las autoridades correspondientes de la Secretaría de Salud de Matamoros, Tamaulipas; se procedió a identificar a los adultos mayores a través de las visitas domiciliarias, contexto en el que se les invitó a participar y se les explicó el objetivo del estudio, sus fines e implicaciones; se aseguró la confidencialidad de los datos y se solicitó su autorización a través de la firma del consentimiento informado tal y como se establece en la Declaración de Helsinki; se aplicó el instrumento en un espacio tranquilo y libre de distractores. Los datos se procesaron y analizaron en el paquete estadístico SPSS, versión 21 para Windows. Se utilizó estadística descriptiva y se aplicó la Prueba de Kolmogorov Smirnov con corrección de Lilliefors, debido a que no se encontró distribución normal en las variables se utilizó estadística no paramétrica con la Prueba de Correlación de Spearman.

\section{RESULTADOS}

Los cuidadores que participaron en el estudio tenían una edad promedio de 39.97 ( $D E=13.12$ ) años, con una escolaridad de $9.86(D E=3.65)$ años. El 73.3\% $(f=63)$ mujeres, el 69.8\% $(f=60)$ con pareja marital, el 68.6\% $(f=59)$ de religión católica, el 34.9\% $(f=30)$ refirió ser hija/o del adulto mayor al que cuida y el 29.1\% $(f=25)$ realiza actividades del hogar además de cuidar al adulto mayor (Tabla 1). Los cuidadores indicaron que en promedio dedican $14(D E=7.57)$ horas al cuidado del adulto mayor.

Tabla 1. Descripción de cuidadores de adultos mayores dependientes

\begin{tabular}{lcc}
\hline Variable & f & $\%$ \\
\hline Religión & 59 & 68.6 \\
\hline Católica & 16 & 18.6 \\
\hline Cristiana & 2 & 2.3 \\
\hline Testigo de Jehová & 5 & 5.8 \\
\hline Otro & 4 & 4.7 \\
\hline Ninguna & & \\
\hline Parentesco con el adulto mayor & 47 & 54.7 \\
\hline Hija/o & 16 & 18.6 \\
\hline Nieta/o & 7 & 8.1 \\
\hline Sobrina/o & 3 & 3.5 \\
\hline Esposa/o & 13 & 15.1 \\
\hline Otro & & \\
\hline Ocupación & 22 & 25.6 \\
\hline Obrero & 25 & 29.1 \\
\hline Hogar & 7 & 8.1 \\
\hline Estudiante & 3 & 33.7 \\
\hline Desempleado & 29 & \\
\hline Otro & & \\
\hline
\end{tabular}


El 64\% $(f=55)$ de los adultos mayores tenían dependencia severa, el 23.3\% $(f=20)$ dependencia moderada, el 11.6\% $(f=10)$ dependencia total y el 1.1\% $(f=1)$ dependencia escasa. En relación con el funcionamiento familiar, el $74.4 \%(f=64)$ de los cuidadores percibieron una funcionalidad familiar normal, el 22.1\% $(f=19)$ disfunción moderada y el 3.5\% $(f=3)$ disfunción grave. El 34.9\% $(f=30)$ de los cuidadores resultó sin sobrecarga, el $32.6 \%(f=28)$ con sobrecarga leve y el $32.6 \%(f=28)$ con sobrecarga intensa.

En cuanto a la calidad de vida de los cuidadores, el 70.9\% $(f=61)$ de manera general puntuó su calidad de vida como aceptable, el 20.9\% ( $f=18)$ alta y el 8.1\% $(f=7)$ deficiente. El 54.7\% ( $f=47)$ presentó una satisfacción aceptable de su estado de salud, el 29.9\% $(f=25)$ satisfacción alta y el 16.3\% ( $f=14)$ satisfacción deficiente. En la dimensión de salud física se encontró una media de 56.35 ( $D E=11.78)$, en la salud psicológica de 56.78 ( $D E=12.97)$, en las relaciones sociales de $45.54(D E=14.83)$ y en el medio ambiente $51.30(D E=9.05)$ (Tabla 2).

Tabla 2. Descripción de la dependencia del adulto mayor, funcionamiento familiar, sobrecarga y dimensiones de la calidad de vida del cuidador

\begin{tabular}{lcccc}
\hline Variable & Min & Max & Media & DE \\
\hline Dependencia del Adulto Mayor & 0 & 95 & 50.17 & 21.65 \\
\hline Funcionamiento familiar & 1 & 10 & 7.76 & 2.17 \\
\hline Sobrecarga del cuidador & 30 & 84 & 51.20 & 11.48 \\
\hline Salud Física & 21.43 & 82.14 & 56.35 & 11.78 \\
\hline Salud Psicológica & 25.00 & 95.83 & 56.78 & 12.97 \\
\hline Relaciones Sociales & 16.67 & 75.00 & 45.54 & 14.83 \\
\hline Medio Ambiente & 31.25 & 75.00 & 51.30 & 9.05 \\
\hline
\end{tabular}

Con la Prueba de Correlación de Spearman se observó que, a mayor funcionamiento familiar, mayor calidad de vida en las dimensiones de salud física ( $p=.001)$, salud psicológica $(p=.000)$, relaciones sociales ( $p=.000$ ) y medio ambiente ( $p=.000$ ). A mayor sobrecarga, menor calidad de vida en las dimensiones de salud física $(\mathrm{p}=.000)$, salud psicológica $(p=.000)$, relaciones sociales $(p=.000)$ y medio ambiente $(p=.000)$. Adicionalmente, se hizo un análisis en busca de la relación entre la dependencia funcional del adulto mayor y el tiempo en horas que el cuidador dedica al cuidado con la calidad de vida, sin embargo; no se encontró relación estadísticamente significativa entre estas variables (Tabla 3).

Tabla 3. Correlación* entre funcionamiento familiar, sobrecarga del cuidador, dependencia del adulto mayor y las dimensiones que evalúan la calidad de vida del cuidador

\begin{tabular}{|c|c|c|c|c|c|c|c|c|}
\hline \multirow[b]{2}{*}{ Variable } & \multicolumn{2}{|c|}{ Salud Física } & \multicolumn{2}{|c|}{ Salud Psicológica } & \multicolumn{2}{|c|}{ Relaciones Sociales } & \multicolumn{2}{|c|}{ Medio Ambiente } \\
\hline & rs & $p$ & rs & $p$ & rs & $p$ & rs & $p$ \\
\hline Funcionamiento familiar & .358 & .001 & .441 & .000 & .419 & .000 & .322 & .000 \\
\hline Sobrecarga del cuidador & -.437 & .000 & -.593 & .000 & -.350 & .001 & -.478 & .000 \\
\hline Dependencia del Adulto Mayor & .157 & .148 & .056 & .609 & -.015 & .889 & .060 & .582 \\
\hline Horas al día al cuidado & -.049 & .656 & .961 & .579 & -.004 & .970 & -.024 & .828 \\
\hline
\end{tabular}

${ }^{*}$ Correlación de Spearman

\section{DISCUSIÓN}

El predominio del género femenino en los cuidadores estudiados, coincide con caracterizaciones realizadas en países como México y Colombia ${ }^{26}$, lo que podría confirmar lo descrito en la literatura 
consultada en donde se identifica la labor de cuidado como una actividad asociada a la mujer. No obstante; el tercio de cuidadores que la presente investigación caracterizó, da cuenta del aumento de la participación masculina en el cuidado de las personas en situación de cronicidad o dependencia funcional, lo cual está siendo descrito por algunos autores ${ }^{27}$.

En lo que respecta a datos demográficos y asociados a la labor de cuidador encontrados en la presente investigación, tales como estado civil casado, edad promedio de 39.9 años, tener un vínculo familiar con el receptor de cuidado, profesar algún tipo de religión y dedicar en promedio 14 horas al cuidado de la persona; fueron situaciones con las que se encontró similitud con resultados publicados en otros países de América Latina ${ }^{26-28}$.

En relación con el funcionamiento familiar, tres cuartas partes de los cuidadores percibieron una funcionalidad familiar normal, en este sentido los hallazgos de Sánchez y cols. ${ }^{17}$ y Cerquera y cols. ${ }^{29}$ son cercanos en algunos puntos porcentuales con la presente investigación, pues ellos encontraron, que $62.5 \%$ y $64 \%$ respectivamente de los cuidadores percibían una funcionalidad familiar normal. En contraste con lo anterior, en una investigación realizada en Cochabamba, Bolivia, se encontró que el $43 \%$ de los cuidadores presentaban una disfuncionalidad familiar severa y el 19\% disfuncionalidad moderada ${ }^{30}$. En consecuencia, tenemos que los datos aquí encontrados son congruentes con los reportes de la mayoría de la literatura consultada, la cual documenta una funcionalidad familiar normal percibida tanto por los cuidadores, como por la persona cuidada ${ }^{18,31}$.

Cabe destacar que poco menos de tres cuartas partes de los sujetos que participaron reportaron tener pareja y posiblemente una vida en familia, lo que significa un involucramiento emocional y físico por parte de sus integrantes, lo cual posiblemente contribuyó en la percepción de una funcionalidad familiar normal o adecuada. Además, el mexicano tiende a desarrollar de la mejor forma relaciones personales y trabajo en equipo para alcanzar objetivos, es decir esta cultura conlleva el cuidado al interior de la familia ${ }^{32}$.

En cuanto al nivel de sobrecarga, 34.9\% de los cuidadores resultó sin sobrecarga, $32.6 \%$ con sobrecarga leve y $32.6 \%$ con sobrecarga intensa. En este sentido, investigaciones son congruentes con los datos aquí descritos ya que identificaron algún nivel de sobrecarga al momento de evaluar esta condición con la escala de Zarit. En Colombia, Silva y cols. ${ }^{14}$ encontraron que el $18.1 \%$ de los cuidadores presentó algún tipo de sobrecarga; así mismo otros autores identificaron que $23.7 \%$ de los cuidadores presenta una sobrecarga severa, $27.3 \%$ sobrecarga leve y $49 \%$ no presentan sobrecarga ${ }^{33}$. De igual forma en México, Palome y cols. ${ }^{15}$ al estudiar el nivel de sobrecarga, encontraron que 56.2\% presentan en alguna medida el síndrome de sobrecarga.

Por otra parte, en lo concerniente a la evaluación que hicieron los cuidadores de su percepción de calidad; de manera general 70.9\% de los cuidadores puntuó su calidad de vida como aceptable. Cabe señalar que muchos cuidadores consideran que su salud es normal, sin embargo; su condición tiende a cambiar con el paso de los años debido al proceso de cuidar al adulto mayor, llegando entonces a afectar la salud de la persona ${ }^{12}$.

Al respecto, piezas investigativas consultadas reportan diversos hallazgos en relación con la valoración que hace el cuidador respecto a su calidad de vida y coinciden con la evaluación que hicieron los cuidadores objeto del presente estudio, ya que estas investigaciones encontraron valoraciones de la calidad como aceptable o en nivel medio ${ }^{13,34}$.

Contrario a esto, Delgado y cols..$^{22}$ encontraron que cuidadores de un grupo de personas sometidas a hemodiálisis evaluaban su calidad de vida como mala y muy mala; de igual forma, en un estudio sobre calidad de vida en cuidadores informales de personas con enfermedades crónicas, 
concluyeron que los cuidadores presentan un deterioro general de su calidad de vida con déficit marcado en la esfera relacional ${ }^{35}$.

Al abordar aspectos relacionados con el fenómeno de ser cuidador de una persona con algún grado de dependencia, se llega a una pluralidad de condicionantes de este al encontrar el concepto de funcionalidad familiar, descrita por algunos autores como un predictor de la salud mental de los cuidadores $^{36}$; misma que ha sido asociada con otras variables, como lo son la calidad de vida y la sobrecarga, que permiten vislumbrar la importancia de la misma.

En este sentido, la presente investigación buscó relacionar la función familiar con la calidad de vida, al igual que la sobrecarga con esta última variable; lo que arrojó. que a mayor sobrecarga, menor calidad de vida del cuidador en todas las dimensiones. Este resultado confirmado por lo reportado en otro estudio ${ }^{14}$ al evaluar estas variables en un grupo de cuidadores, se observó que el desgaste de la calidad de vida está relacionado con la sobrecarga en el cuidado del adulto mayor. De igual forma, Piñánez y otros ${ }^{37}$ identificaron relación estadística significativa entre niveles altos de sobrecarga y un impacto en la calidad de vida, representado por síntomas de depresión en los cuidadores participantes.

Luego de evaluar el tipo de relación entre la funcionalidad familiar y niveles de calidad de vida, se obtuvo que a mayor funcionamiento familiar, mayor calidad de vida del cuidador, dichos resultados son respaldados por los obtenidos por autores como Troncoso-Pantoja y Soto-López ${ }^{38}$, quienes concluyeron luego de estudiar la dinámica familiar de familias con adultos mayores dependientes; que aquellos cuidadores y adultos mayores pertenecientes a familias con una mejor dinámica interna, disfrutan de una mejor calidad de vida, conclusión que coincide con la expresada por ValdezHuirache y Álvarez-Bocanegra ${ }^{39}$, quienes afirmaron posteriormente a su investigación, que a mayor apoyo social mejor calidad de vida en adultos mayores y cuidadores.

\section{CONCLUSIONES}

Se observó que, a mayor funcionamiento familiar, mayor calidad de vida del cuidador y a mayor sobrecarga menor calidad de vida en todas las dimensiones. Adicionalmente se hizo un análisis en busca de la relación entre la dependencia funcional del adulto mayor y el tiempo en horas que el cuidador dedica al cuidado con la calidad de vida, sin embargo; no se encontró relación estadísticamente significativa entre estas variables.

El abordaje de fenómenos relacionados con el cuidador de la persona adulta mayor, sea cual fuese su condición de dependencia, se convierte en una necesidad, toda vez que se desconoce en la mayoría de los casos las condiciones en las que se da este cuidado. En este sentido, aunque se reconoce que el muestreo y tamaño de muestra constituyen una limitante para la generalización de los resultados; los hallazgos descritos se convierten en un escenario disciplinar que plantea la posibilidad de diseñar estrategias de intervención enfocadas al mejoramiento de la calidad de vida del cuidador, con repercusión en la calidad del cuidado que este brinda.

Además, el estudio de este tipo de fenómenos enmarca su contribución al campo de estudio de enfermería, mediante aportes sociales y disciplinares que surgen a través de resultados empíricos que permiten el rediseño o formulación de planes de intervención colectivos y políticas publicas orientadas a la atención integral de las diadas de enfermedades crónicas.

Por último, cabe destacar que estos resultados vislumbran un panorama que enriquece la practica disciplinar, toda vez que permite conocer el comportamiento de variables de interés a la hora de brindar atención a estos colectivos 


\section{RESPONSABILIDADES ÉTICAS}

Protección de personas y animales. Los autores declaran que para esta investigación no se han realizado experimentos en seres humanos ni en animales.

Confidencialidad de los datos. En este artículo no aparecen datos confidenciales de los participantes. Derecho a la privacidad y consentimiento informado. Se solicitó el consentimiento informado previo al inicio de la investigación conforme lo marca el reglamento en materia de investigación en salud.

Financiamiento. No se recibió ningún tipo de financiamiento para la presente investigación.

Conflicto de intereses. Los autores declaran no tener conflicto de intereses.

\section{REFERENCIAS}

1. Instituto Nacional de Estadística y Geografía (INEGI). Estadísticas al propósito del día internacional de las personas de edad. México: INEGI; 2018. https://bit.ly/2qfJgLW

2. Consejo Nacional de Población. La situación Demográfica de México 2011. México: CONAPO; 2011.

3. Organización Mundial de la salud (OMS). Envejecimiento y salud. Ginebra: OMS; 2015. https://bit.ly/1RlXbWA

4. Duran-Badillo T, Domínguez-Chávez CJ, Hernández-Cortés PL, Félix-Alemán A, Cruz-Quevedo JE, Alonso-Castillo MM. Dejar de ser o hacer: significado de dependencia funcional para el adulto mayor. Acta Univ. 2018; 28(3): 40-46. http://dx.doi.org/10.15174/au.2018.1614

5. Flores E, Rivas E, Seguel F. Nivel de sobrecarga en el desempeño del rol del cuidador familiar de adulto mayor con dependencia severa. Cienc. Enferm. 2012; 18 (1): 29-41.

http://dx.doi.org/10.4067/So717-95532012000100004

6. Villegas-Ceja MH, Aguilar-Chagoyán MA, Espericueta-Medina M, Medina-Sánchez MJ, Luján-López ME. Desempeño del rol de cuidador primario y eficiencia del programa Atención Hospitalaria a Domicilio. Rev Enferm Inst Mex Seguro Soc. 2014; 22(1):25-32. https://bit.ly/2MLswUp

7. Zarit SH, Bottigi K, Gaugler JE. Stress and caregivers. En: Fink G. (Ed). Encyclopedia of Stress. 2nd Edition. San Diego, CA: Academic Press; 2007. p. 416-18.

8. Aguilar-Gutierrez AE, Jiménez-Reyes J, Álvarez-Aguirre A, Sánchez-Perales M, Ortega-Jiménez M. Sobrecarga del cuidador principal del adulto mayor con enfermedad alzhéimer. Epistemus. 2016; 21 (10): 30-6. https://bit.ly/2ISwb1t

9. Ares-Muzio P. Psicología de la Familia. Libro en formato digital. La Habana: Facultad de Psicología, Universidad de la Habana; 2007.

10. Suárez-Cuba MA, Alcalá-Espinoza M. APGAR familiar: una herramienta para detectar disfunción familiar. Rev Méd la Paz. 2014; 20 (1): 53-7. https://bit.ly/2MLnnf1

11. Casanova-Rodas L, Rascón-Gasca ML, Alcántara-Chabelas H, Soriano-Rodríguez A. Apoyo social y funcionalidad familiar en personas con trastorno mental. Salud Ment. 2014; 37(5): 443-8. https://bit.ly/31nuGyK

12. Ferraz K, Silva RN, Pereira R. Quality of life of relative caregivers of elderly, Texto Context-Enferm. 2014; 23(3): 600-8 https://bit.ly/2MOXwXS

13. Galvis-López, CR, Aponte-Garzón LH, Pinzón-Rocha ML. Percepción de la calidad de vida de cuidadores y pacientes asistentes a un programa de crónicos, Villavicencio, Colombia. Aquichan. 2016; 16 (1): 104-15. http://dx.doi.org/1052947aqui.2016.16.1.11 
14. Silva Fhon JR, Gonzales-Janampa JT, Mas-Huaman T, Marques S, Pertezani Rodrigues RA. Sobrecarga y calidad de vida del cuidador principal del adulto mayor. Av. enferm. 2016; 34(3): 251-8.

http://dx.doi.org/10.15446/av.enferm.v34n3.58704

15. Palome-Vega G, Gallegos-Torres RM, Xeque-Morales AS, Juárez-Lira A, Perea-Ortíz MG. Nivel de sobrecarga en el cuidador principal del paciente crónico hospitalizado. Ciencia@uaqro.2014; 1-10. https://bit.ly/2F430FN

16. Rodríguez-Medina RM, Landeros-Pérez ME. Sobrecarga del agente de cuidado dependiente y su relación con la dependencia funcional del adulto mayor. Enferm univ. 2014; 11(3): 87-93. https://doi.org/10.1016/S1665-7063(14)72671-5

17. Sánchez-Martínez RT, Molina-Cardona EM, Gómez-Ortega OR. Intervenciones de enfermería para disminuir la sobrecarga en cuidadores: un estudio piloto. Rev cuid. 2016; 7(1): 1171-84. http://dx.doi.org/10.15649/cuidarte.v711.251

18. Padrón-Quezada FR, Peña-Cordero S, Salazar-Torres K. Funcionalidad familiar asociado al cuidado del adulto mayor con hipertensión arterial crónica; Comunidad Coyoctor. Rev. Latinoam. Hipertens. 2017; 12(3): 79-82. https://bit.ly/35yIPwq

19. Cid-Ruzafa J, Damián-Moreno J. Valoración de la discapacidad física: el índice de Barthel. Rev esp salud pública. 1997; 71(2), 127-37. https://bit.ly/2lWpujU

20. Shah S, Vanclay F, Cooper B. Improving the sensitivity of the Barthel Index for stroke rehabilitation. J Clin Epidem. 1989; 42(8): 703-9. https://doi.org/10.1016/0895-4356(89)90065-6

21. Montero-Pardo X, Jurado-Cárdenas S, Valencia-Cruz A, Méndez-Venegas J, Mora-Magaña I. Escala de carga del cuidador de Zarit: Evidencia de validez en México. Psicooncología. 2014, 11(1): 71-85. https://doi.org/10.5209/rev_PSIC.2014.v11.n1.44918

22. Delgado-Quiñones EG, Barajas-Gómez TDJ, Uriostegui-Espiritu L, López-López V. Sobrecarga y percepción de la calidad de vida relacionada con el cuidador primario del paciente hemodializado. Rev Cubana Med Gen Integr. 2016; 32(4), 1-9. https://bit.ly/2VOg2jo

23. Flores-Herrera BI, Castillo-Muraira Y, Ponce-Martínez D, Miranda-Posadas C, Peralta-Cerda EG, Durán-Badillo T. Percepción de los adultos mayores acerca de su calidad de vida. Una perspectiva desde el contexto familiar. Rev. enferm. Inst. Mex. Seguro Soc. 2018; 26(2): 83-8. https://bit.ly/2nSGlIf

24. Torres M, Quezada M, Rioseco R, Ducci ME. Calidad de vida de adultos mayores pobres de viviendas básicas: Estudio comparativo mediante uso de WHOQ_oL-BREF. Rev méd Chile. 2008; 136(3): 325-33. http://dx.doi.org/10.4067/S0034-98872008000300007

25. Skevington SM, Lofty M. O'Connell KA, WHOQOL Group. The World Health Organization's WHOOOLBREF quality of life assessment: Psychometric properties and results of the international field trial A Report from the WHOQOL Group. Qual Life Res. 2004; 13(2):299-310. https://doi.org/10.1023/B:OURE.0000018486.91360.00

26. Barreto-Osorio R, Coral-Ibarra R, Campos MS, Gallardo-Solarte K, Ortiz VT. Cuidadores y cuidadoras familiares de personas con enfermedad crónica en Colombia: más similitudes que diferencias. Salud Uninorte. 2015; 31(2): 255-65. http://dx.doi.org/10.14482/sun.30.1.4309

27. Sánchez-Herrera B, Carrillo-González GM, Corredor-Parra LR. Caracterización y carga del cuidado en hombres cuidadores familiares de personas con enfermedad crónica en Colombia. Arch Med (Manizales) 2014; 14 (2), 219-30. https://doi.org/10.30554/archmed.14.2.299.2014

28. Romero-Massa E, Rodríguez-Castilla J, Pereira-Díaz B. Sobrecarga y calidad de vida percibida en cuidadores familiares de pacientes renales. Rev. Cubana Enfermer. 2015; 31(4). https://bit.ly/20OfLOC 
29. Cerquera-Córdoba AM, Matajira-Camacho J, Pabón-Poches DK. Caracterización de una muestra de cuidadores formales de pacientes con trastorno neurocognitivo mayor en Bucaramanga. Rev. virtual. univ. catol. norte. 2016; (47): 4-19. https://bit.ly/2BwlbDf

30. Mavric-Arebalo K, Carpio-Deheza G. Calidad de vida y funcionalidad familiar del cuidador del paciente con enfermedad de alzheimer. Revista Médico-Científica “Luz y Vida”. 2017; 8 (1): 20-6. https://bit.ly/2VOmwxL

31. Puigvert-Vilalta C. Percepción de la satisfacción de la función familiar que tienen los pacientes de diálisis peritoneal. Enferm Nefrol. 2013; 16(Supl.1), 28-9.https://dx.doi.org/10.4321/S2254-28842013000500013

32. Gutiérrez R, Díaz KY, Román RP. El concepto de familia en México: una revisión desde la mirada antropológica y demográfica. Ciencia ergo sum, 2016; 23(3), 219-228. https://bit.ly/2NJo7QO

33. Pinzón E, Carrillo G. Carga del cuidado y calidad de vida en cuidadores familiares de personas con enfermedad respiratoria crónica. Rev Fac Nac Salud Pública. 2016; 34 (2): 193-201.

https://doi.org/10.17533/udea.rfnsp.v34n2ao8

34. Covarrubias L, Andrade RMG. Calidad de Vida de Cuidadores de Pacientes Hospitalizados, nivel de dependencia y red de apoyo. Index Enferm. 2012; 21(3): 131-5. https://dx.doi.org/10.4321/S1132-12962012000200005

35. Ávila-Toscano JH, Vergara-Mercado M. Calidad de vida en cuidadores informales de personas con enfermedades crónicas. Aquichan. 2014; 14(3): 417-29. https://doi.org/10.5294/aqui.2014.14.3.11

36. Pérez-Peñaranda A, García-Ortiz L, Rodríguez-Sánchez E, Losada Baltar A, Porras-Santos N, GómezMarcos MÁ. Función familiar y salud mental del cuidador de familiares con dependencia. Aten. prim. (Barc., Internet). 2009; 41(11): 621-8. https://doi.org/10.1016/j.aprim.2009.03.005

37. Piñánez-García MC, Re-Dominguez ML, Núñez-Cantero AM. Sobrecarga en cuidadores principales de Adultos Mayores. Rev. salud pública parag. 2016; 6(2): 10-15. https://bit.ly/2oGXx46

38. Troncoso-Pantoja C, Soto-López N. Funcionalidad familiar, autovalencia y bienestar psicosocial de adultos mayores. Horiz Méd. 2018; 18(1):23-8. https://dx.doi.org/10.24265/horizmed.2018.v18n1.04

39. Valdez-Huirache MG, Álvarez-Bocanegra C. Calidad de vida y apoyo familiar en adultos mayores adscritos a una unidad de medicina familiar. Horiz sanitario. 2018; 17(2):113-21.

http://dx.doi.org/10.19136/hs.a17n2.1988 